Entropy driven spontaneous formation of highly porous films from polymer-nanoparticle composites

This article has been downloaded from IOPscience. Please scroll down to see the full text article.

2009 Nanotechnology 20425602

(http://iopscience.iop.org/0957-4484/20/42/425602)

View the table of contents for this issue, or go to the journal homepage for more

Download details:

IP Address: 128.206.162.204

The article was downloaded on 10/08/2010 at 20:58

Please note that terms and conditions apply. 


\title{
Entropy driven spontaneous formation of highly porous films from polymer-nanoparticle composites
}

\author{
Venumadhav Korampally ${ }^{1}$, Minseong Yun ${ }^{1}$, \\ Thiruvengadathan Rajagopalan ${ }^{1}$, Purnendu K Dasgupta ${ }^{2}$, \\ Keshab Gangopadhyay ${ }^{1}$ and Shubhra Gangopadhyay ${ }^{1,3}$ \\ ${ }^{1}$ Department of Electrical Engineering, University of Missouri-Columbia, 349 Engineering \\ Building West, Columbia, MO 65211, USA \\ ${ }^{2}$ Department of Chemistry and Biochemistry, University of Texas at Arlington, 340 A CPB, \\ Arlington, TX 76019, USA \\ E-mail: Gangopadhyays@missouri.edu
}

Received 28 April 2009, in final form 20 August 2009

Published 25 September 2009

Online at stacks.iop.org/Nano/20/425602

\begin{abstract}
Nanoporous materials have become indispensable in many fields ranging from photonics, catalysis and semiconductor processing to biosensor infrastructure. Rapid and energy efficient process fabrication of these materials is, however, nontrivial. In this communication, we describe a simple method for the rapid fabrication of these materials from colloidal dispersions of Polymethyl Silsesquioxane nanoparticles. Nanoparticle-polymer composites above the decomposition temperature of the polymer are examined and the entropic gain experienced by the nanoparticles in this rubric is harnessed to fabricate novel highly porous films composed of nanoparticles. Optically smooth, hydrophobic films with low refractive indices (as low as 1.048) and high surface areas (as high as $1325 \mathrm{~m}^{2} \mathrm{~g}^{-1}$ ) have been achieved with this approach. In this communication we address the behavior of such systems that are both temperature and substrate surface energy dependent. The method is applicable, in principle, to a variety of nanoparticle-polymer systems to fabricate custom nanoporous materials.
\end{abstract}

(Some figures in this article are in colour only in the electronic version)

\section{Introduction}

The ability to control the nanostructure of materials is increasingly becoming important to realize materials with improved optical, mechanical and electrical properties. Over the last decade, nanoporous materials have become ubiquitous in sensor technologies [11], drug delivery [27], low $k$ dielectrics $[19,16]$, catalysis [9] and photonics [3, 26], to name a few. Highly porous films deposited from silica based solgel precursors [2], surfactant templated sol-gel mesoporous films [2, 4, 32] and organosilicate nanoporous films formed via sacrificial porogens [16] are among the most widely used materials for these applications. The fabrication of these films with good control over their properties is nontrivial. Tight

\footnotetext{
${ }^{3}$ Author to whom any correspondence should be addressed.
}

control on the relative humidity of the environment and the ageing conditions is crucial to achieve good quality sol-gel films [12]. These films, in addition, are highly susceptible to moisture absorption unless post-treatment is performed to render them hydrophobic. Surfactant templates and sacrificial porogens typically permit a greater control on the porosity and pore size. High quality films with pore sizes $<10 \mathrm{~nm}$ have been reported for use as low $k$ dielectrics [16] and as antireflection coatings [3]. However, these films, with their rigid crosslinked networks, frequently suffer from large residual thermal stresses that initiate cracking and buckling, especially when thick films are needed [20,21]. Highly controlled reaction parameters and slow curing times are required during their fabrication.

The versatility of these materials coupled with the growing demand is spawning the need for a novel rapid energy efficient fabrication methodology. Polymer-nanoparticle composites 


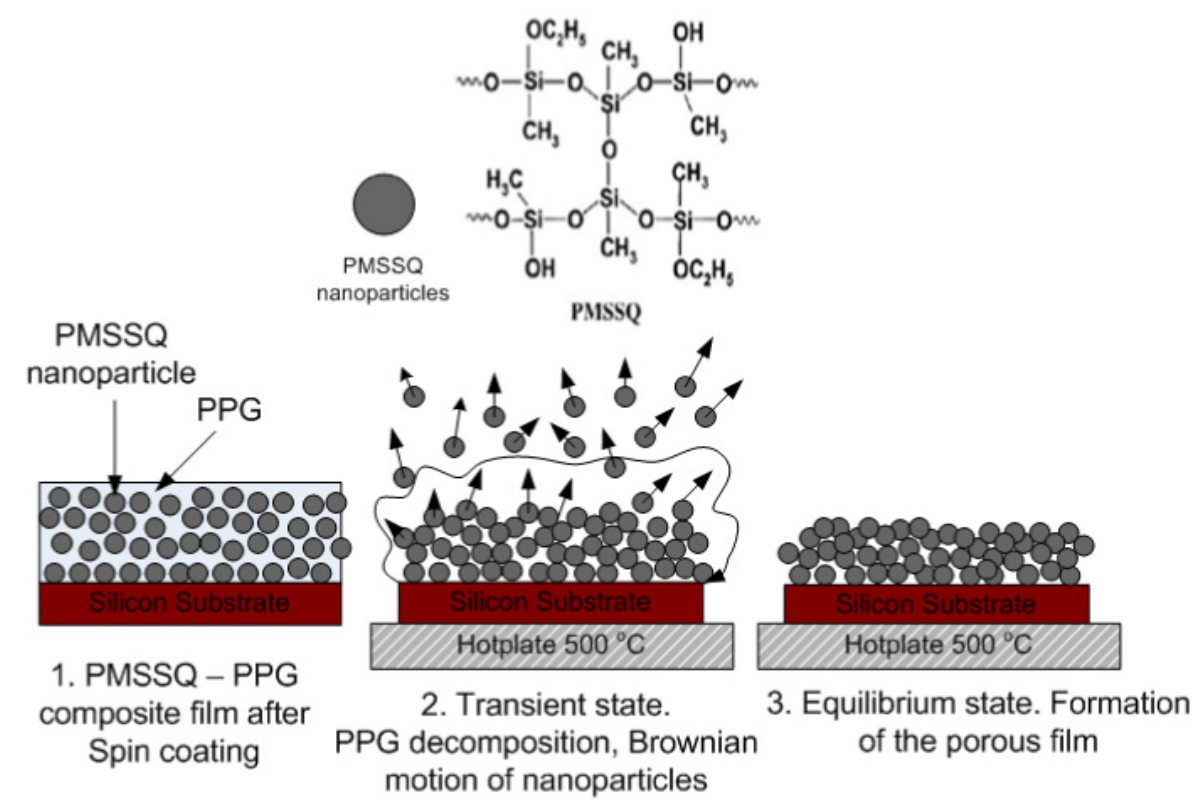

Figure 1. Schematic of the PMSSQ-PPG system under high temperature curing conditions.

are of considerable interest recently as they offer a novel means to fabricate multifunctional materials. The study of the self-organization of nanostructures through the interplay between entropic and enthalpic interactions is especially relevant $[17,13,5,30,14,7,18,1,29]$. It has been demonstrated that nanoparticles could be entropically driven to self-assemble into polymer-nanoparticle multilayers by controlling the nature of the ligands on the nanoparticle surfaces [14]. Entropic and enthalpic interactions between the nanoparticles and the polymer matrix have been exploited to fabricate coatings that are self-healing [30,7].

Nanoparticle-polymer composites heated above the polymer decomposition temperature offer an interesting unexplored avenue. The increase in the entropy of the system when heated above the decomposition temperature of the polymer provides a degree of mobility to the initially confined nanoparticles. We show that with nanoparticles bearing crosslinkable/reactive functional groups, this increased entropy translates to a random movement and collisions between the nanoparticles, the interparticle crosslinking finally driving the system to a new equilibrium. This behavior can be harnessed to rapidly fabricate nanoporous films with controlled optical and physical properties. As the film formation is essentially entropy driven, it is reasonable to expect that the magnitude of the thermal input will play a critical role in determining the final equilibrium state. In addition, interactions between the nanoparticles and the substrate surface may also control nanoparticle mobility and determine the final state of the system.

In this work, we explore the behavior of such a system under two temperature regimes, above and below the decomposition temperature of the polymer, and with different substrates distinguished by different surface energies. Our system essentially consists of functionalized nanoparticles that could be thermally initiated to undergo interparticle/intraparticle crosslinking, dispersed in a polymer, the thermal lability of which is subsequently brought into play. Polymethyl silsesquioxane (PMSSQ) nanoparticles dispersed in polypropylene glycol (PPG) is the presently chosen example: PMSSQ is the most studied starting material for nanoporous dielectrics $[31,23,19,25,15,16]$; it is inexpensive and PMSSQ based films are naturally hydrophobic, thus minimizing degradation due to moisture absorption. Figure 1 gives a schematic of our system subjected to high temperatures. High temperature here implies that the system is subjected to temperatures above the decomposition temperature of PPG $\left(>200^{\circ} \mathrm{C}\right)$.

\section{Materials and methods}

Commercially available PMSSQ was purchased from Techneglas, Inc. (GR650F) and used for the preparation of PMMSQ nanoparticles. The nanoparticles were obtained by dissolving PMSSQ in a good solvent (propylene glycol methyl ether acetate, PGMEA, 98\% Sigma-Aldrich) and initiating polymer collapse $[33,34,8,24]$ by the addition of a low molecular weight PPG (number average molecular weight $\left(M_{n}\right) 425$, Sigma-Aldrich). Selective evaporation of PGMEA occurs during the film deposition step, enriching the concentration of PPG with respect to PMSSQ. The amphiphilic nature of the PPG molecules ensures good miscibility of the two polymers, while the relatively hydrophilic nature of the polymer forces the hydrophobic PMSSQ molecules to assume a compact globular form. Details on the formation mechanism of the particles will be discussed elsewhere. The PMSSQ to PPG mass ratio was maintained at $50 \%$ for all the experiments and all films were deposited from a PMSSQ:PPG:PGMEA solution with wt\% composition of 1:1:2 respectively onto hydrogen passivated silicon substrates unless otherwise mentioned. Hydrogen passivation was achieved by dipping the silicon substrates in a 

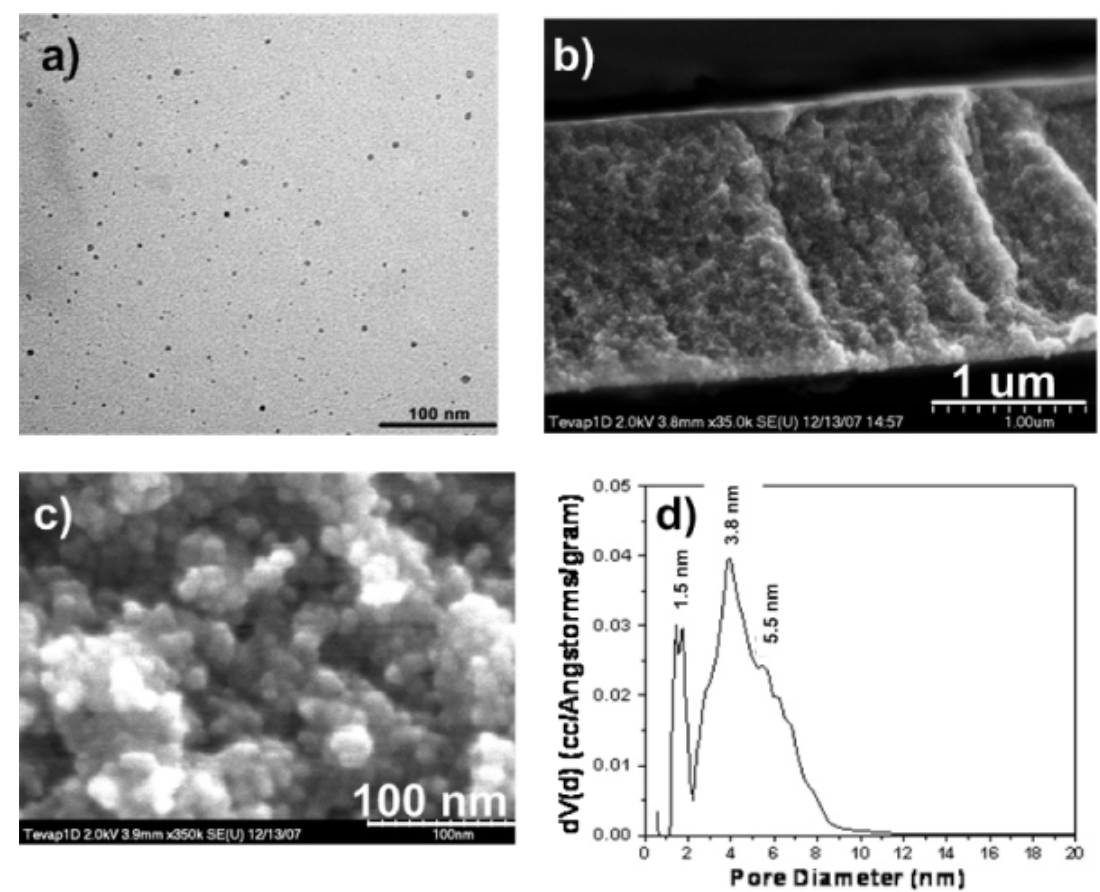

Figure 2. (a) TEM of the PMSSQ nanoparticles used in this study, (b) and (c) SEM of the film cross section at different magnifications. The films were deposited on $\mathrm{H}$-passivated silicon substrates followed by a rapid thermal curing/calcination at $500^{\circ} \mathrm{C} / 5 \mathrm{~min}$ (d) pore size distribution of the films obtained through nitrogen adsorption-desorption measurements.

1:10 HF: DI water solution for $10 \mathrm{~s}$ immediately prior to film deposition. Film deposition was done through spin coating at $3000 \mathrm{rpm}$ for $30 \mathrm{~s}$. Following the spin coating process, the obtained films were immediately subjected to a high temperature treatment step by placing the substrates on a pre-heated hot plate. Temperatures ranging from 200 to $600{ }^{\circ} \mathrm{C}$ were used in this study. All the films were cured for $5 \mathrm{~min}$, after which they were removed from the hotplate and allowed to cool down to room temperature.

Optical characterization of these films was performed using variable angle spectroscopic ellipsometry (VASETM, J A Wollam, Inc.) to obtain their thickness and refractive index (measured at $630 \mathrm{~nm}$ wavelength) applying the Cauchy model [22]. Fourier transform infrared spectroscopy of the films was performed using a Thermo Nicolet FTIR system. Cross-sectional scanning electron microscopy (SEM) images of the film were obtained with a field emission Hitachi $\mathrm{S}$ 4700 microscope with acceleration voltages between 3 and $5 \mathrm{kV}$. The films were coated with a thin layer of carbon $(\sim 12 \mathrm{~nm})$ to prevent charging during imaging. For the BET surface area analysis and the pore size measurement, the films were scraped from the substrates with a razor edge and the powder characteristics were measured. Nitrogen adsorptiondesorption isotherms were measured using a Quantachrome Autosorb-1 automated gas sorption system and the surface areas of the samples were computed using the BET method.

For the surface energy dependent system behavior study, surface energies of various substrates were calculated from the static contact angle measurement of diiodomethane, formamide, and deionized (DI) water as outlined in [6]. Substrates used for this study included hydrogen passivated silicon substrates, Piranha treated borosilicate glass substrates, hexamethyldisilazane (HMDS) treated borosilicate glass substrates, and borosilicate glass substrates coated with a proprietary plasma enhanced chemical vapor deposited SiCON film. Piranha treatment of the glass substrates was performed by placing the substrates in a 1:5 volume solution of freshly prepared sulfuric acid (95-98\%,Sigma-Aldrich): hydrogen peroxide (35\%, Sigma-Aldrich) solution for $10 \mathrm{~min}$ followed by rinsing them in a continuous flow of DI water. HMDS vapor treatment of the glass slides was performed by placing freshly Piranha treated glass slides along with a $0.5 \mathrm{ml}$ of HMDS (98\%, Sigma-Aldrich) in a tightly sealed stainless steel chamber. The SS container was later subjected to a heating step inside a convection oven at $80^{\circ} \mathrm{C}$ for $30 \mathrm{~min}$. The substrates were removed after allowing the chamber to cool down to room temperature. Films were deposited from a PMSSQ:PPG:PGMEA solution with a wt\% composition of 1:1:2 respectively through spin coating at $3000 \mathrm{rpm}$ for $30 \mathrm{~s}$. Curing/calcination of the films was performed by placing the coated substrates in a pre-heated furnace at $470{ }^{\circ} \mathrm{C}$ for $5 \mathrm{~min}$, after which they were removed and allowed to cool down to room temperature.

\section{Results and discussion}

Figure 2(a) shows the TEM of the PMSSQ nanoparticles synthesized and used in this study. The particle sizes ranged from 3 to $7 \mathrm{~nm}$ with a mean particle size of $5 \mathrm{~nm}$. Figures 2(b) and (c) show the cross-sectional SEM image of the film obtained by subjecting it to an instantaneous curing/calcination step at $500^{\circ} \mathrm{C}$ for $5 \mathrm{~min}$. It can be observed 


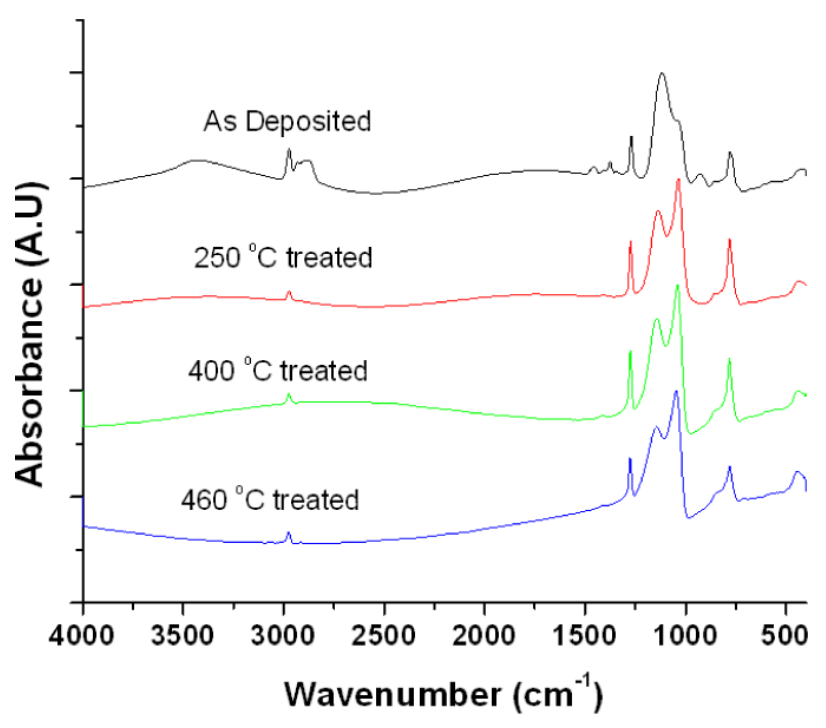

Figure 3. Fourier transform infrared spectra (FTIR) of the films treated at different temperatures.

that the film is relatively thick $(1.8 \mu \mathrm{m}$, figure $2(\mathrm{~b}))$ and the nanoparticulate nature of the films is immediately evident from figure 2(c). Figure 2(d) shows the pore size distribution of these films. These films have both meso. (2-10 $\mathrm{nm})$, and microscale $(<2 \mathrm{~nm})$ porosity and thus exhibit a bimodal pore size distribution. From the SEM image and the pore size distribution plot, it is evident that the nanostructure of these films consists of feature sizes well below the wavelength of light thus resulting in good optical quality. The BET plots are consistent with the microscopically observed highly nanoporous structure, the BET surface area was $1325 \mathrm{~m}^{2} \mathrm{~g}^{-1}$.

Figure 3 gives Fourier transform infrared (FTIR) spectra of the thin films processed at different temperatures. PMSSQ is an organosilicate (empirical formula $\left(\mathrm{CH}_{3}-\mathrm{SiO}_{1.5}\right)_{n}$, ) with $\mathrm{Si}$ and $\mathrm{O}$ atoms located at the alternate corners of a deformed cage and one methyl group attached to each $\mathrm{Si}$ atom. The most intense absorption peaks seen at 1135 and $1035 \mathrm{~cm}^{-1}$ are attributed to a Si-O stretching vibration with caged and bridging configurations respectively [35, 19]. All the absorption features observed in the spectra shown in figure 3 are identified as follows; $2975 \mathrm{~cm}^{-1}-\mathrm{CH}_{3}$ asymmetric stretching mode, $1420 \mathrm{~cm}^{-1}-\mathrm{CH}_{3}$ bending mode, $1275 \mathrm{~cm}^{-1}-\mathrm{Si}-\mathrm{CH}_{3}$ stretching vibration, $845 \mathrm{~cm}^{-1}-\mathrm{Si}-$ $\left(\mathrm{CH}_{3}\right)_{2}, 775 \mathrm{~cm}^{-1}-\mathrm{Si}-\mathrm{CH}_{3}$ bending modes and $450 \mathrm{~cm}^{-1}-\mathrm{Si}-$ $\mathrm{O}$ rocking mode respectively. It is significant to note that no absorption vibrational modes were observed for bonded and free hydroxyl groups at $3000-3600 \mathrm{~cm}^{-1}$ indicating that all the hydroxyl groups present in the precursors were removed during the curing/calcination steps. The disappearance of the $\mathrm{Si}-\mathrm{OH}$ peak (at $930 \mathrm{~cm}^{-1}$ ) with the heat curing step is indicative of the polycondensation reactions of PMSSQ (interparticle and intraparticle) to form $\mathrm{Si}-\mathrm{O}-\mathrm{Si}$ linkages $[19,35]$. The region $2940-2800 \mathrm{~cm}^{-1}$ could be attributed to the $-\mathrm{CH}_{2}$ stretching vibrations from structures present in PPG. The complete disappearance of these peaks is evident in the films treated at higher temperatures $\left(>250^{\circ} \mathrm{C}\right)$. The thickness and refractive index of the films as a function of curing time (temperature $=$

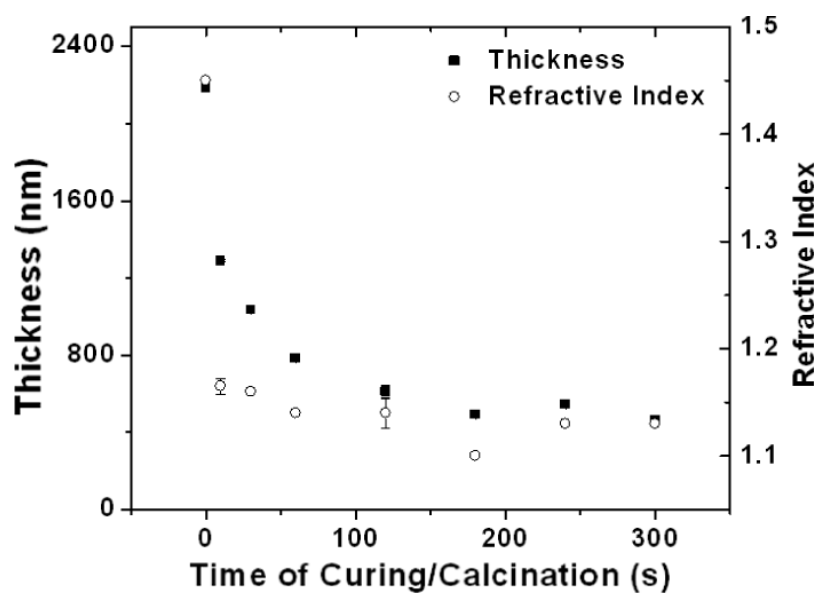

Figure 4. Thickness and refractive index of the films as a function of curing/calcination time (curing/calcination temperature $=530^{\circ} \mathrm{C}$ ).

$530{ }^{\circ} \mathrm{C}$ ) is shown in figure 4 . From the plots, it can be seen that the films tend to reach an equilibrium state within about 3 min of curing/calcination time. Beyond $3 \mathrm{~min}$, not much change in film properties (refractive index and thickness) could be observed. The refractive index as a function of curing/calcination temperature is shown in figure 5(a). A decreasing trend in the refractive indices of these films could be observed with increasing curing/calcination temperatures. Figure 5(b) gives a plot of the thickness of the films as a function of curing/calcination temperature. Figure 6 gives a plot of the refractive index of the films as a function of the PPG loading content with respect to PMSSQ in the precursor solution. Figure 7 shows a picture of the film stored at room temperature $>12 \mathrm{~h}$ before imaging. The magnified image shows the droplets of PPG being expelled from the film.

\subsection{Curing/calcination temperature dependent system behavior}

It is interesting to note that the optical as well as the physical properties of the film depend greatly on the curing/calcination temperature even though PPG decomposition occurs at fairly low temperatures $\left(\sim 200^{\circ} \mathrm{C}\right.$, figure 3$)$. This temperature dependence could be explained by examining the transient states the film passes through before reaching the final equilibrium state.

With the sudden application of heat to the system, PPG decomposition takes place, the expanding gaseous products result in a large increase in the translational entropy/mobility of the PMSSQ nanoparticles as they are no longer confined by the PPG chains. The nanoparticles suspended in the gaseous decomposition products of PPG are in continuous Brownian motion with the average kinetic energy of each particle equal to $3 / 2 \mathrm{kT}\left(\sim 10 \mathrm{~kJ} \mathrm{~mol}^{-1}\right.$ at $\left.500^{\circ} \mathrm{C}\right)$. In addition, the increased internal energy of each individual nanoparticle results in their intraparticle crosslinking, while the random movement and collision between the particles results in their interparticle crosslinking, thereby kinetically arresting the system and driving it to an equilibrium that results in a nanoporous film comprising of nanoparticles. 

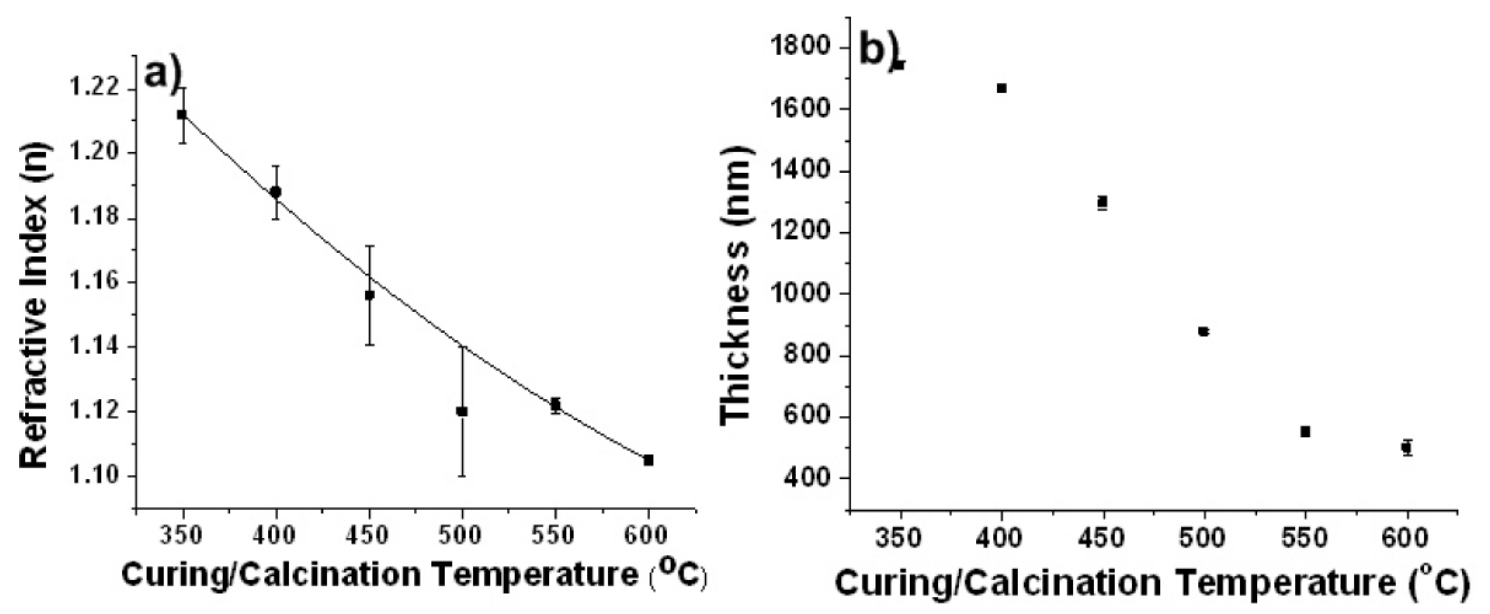

Figure 5. (a) Refractive index of the film as a function of curing/calcination temperature, (b) thickness of the films as a function of the curing/calcination temperature. All the films were prepared on hydrogen terminated silicon substrates.

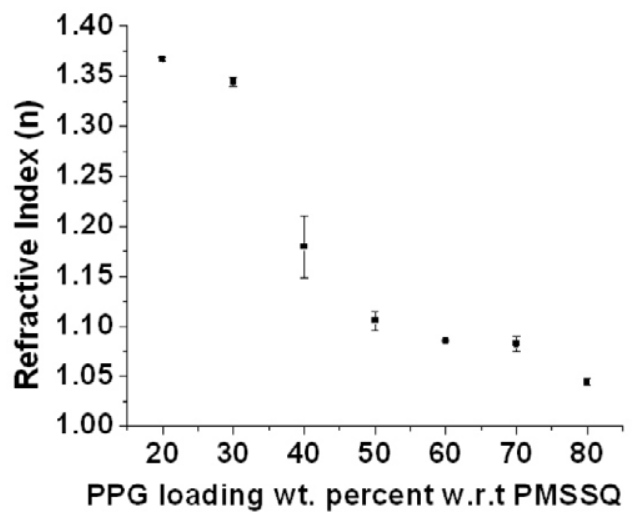

Figure 6. Refractive index as a function of PPG weight per cent w.r.t to PMSSQ nanoparticles. All the films were prepared on hydrogen terminated silicon substrates.

The film thickness continuously decreases with increasing curing/calcination temperatures (figure 5(b)) although the porosity of the film increases as a function of increasing temperature. At any finite temperature, only a fraction of the nanoparticles that undergo random interparticle collisions end up undergoing interparticle crosslinking. It is this interparticle crosslinking that results in reduced mobility and ultimately surface immobilization. Interestingly this runs counter to the notion that the probability of any chemical reaction subject to a finite activation energy (as most polymerization reactions are) will increase with increasing temperature. This is because while the probability of the reaction on a molecular scale must increase with increasing temperature, the relevant kinetic energy is of the entire particle not just the molecules undergoing the reaction. A macroscale equivalent is an adhesive coated ball thrown at a surface; when thrown with a modest force it will stick, but above a certain threshold it will bounce off the surface. At elevated temperatures, a greater fraction of the nanoparticles are thus just expelled into the environment resulting in thinner films (see figures 1 and 5(b)). Optical properties of these films could further be tuned by varying the PPG loading content. It can be observed that ultra-low refractive index films, with refractive indices as low as 1.048 could be achieved following this relatively simple approach. Such low refractive index films obtained by spin coating are unprecedented [28]. It is interesting to note that, even at sufficiently high porogen loadings $(>80 \%)$, the refractive index of films reported using the sacrificial porogen approach is still comparatively high $(\sim 1.13-1.18)[22,10]$. In addition, due to the monolithic structure of these films, it is expected that the optical constants of these films (sacrificial porogen approach) remain constant as a function of calcination temperature provided complete porogen burn out occurs.

At temperatures lower than the decomposition temperature of PPG $\left(<200^{\circ} \mathrm{C}\right)$, PPG chains play an active role in maximizing the system entropy. Segregation of the nanoparticles takes place at these temperatures, whereby the nanoparticles lose their translational entropy. This loss of translational entropy of the nanoparticles is however offset by the gain in the conformational entropy of PPG chains, as there is a far greater entropic penalty imposed in stretching the polymer chains around the nanoparticles to maintain a uniform distribution $[5,14,7]$. This results in the eventual expulsion of PPG and a subsequent densification of the film with time. (See figure 7.)

\subsection{Substrate surface energy dependence}

The surface energy of the substrate also plays a critical role in determining the final state of the system. We have shown above that highly porous films could be obtained when the PMSSQ-PPG composite films are deposited upon hydrogen terminated silicon substrates followed by a high temperature curing/calcination step. Films processed under identical conditions but deposited upon high surface energy substrates resulted in a large loss in the porosity. For example, films deposited on freshly piranha cleaned glass substrates and subjected to the same instantaneous high temperature curing/calcination step resulted in the formation of dense films without any porosity. In the case of high surface energy substrates, we believe that the enthalpic interactions between the nanoparticles and the substrate surface outweighs the translational entropic gain resulting in their segregation and 


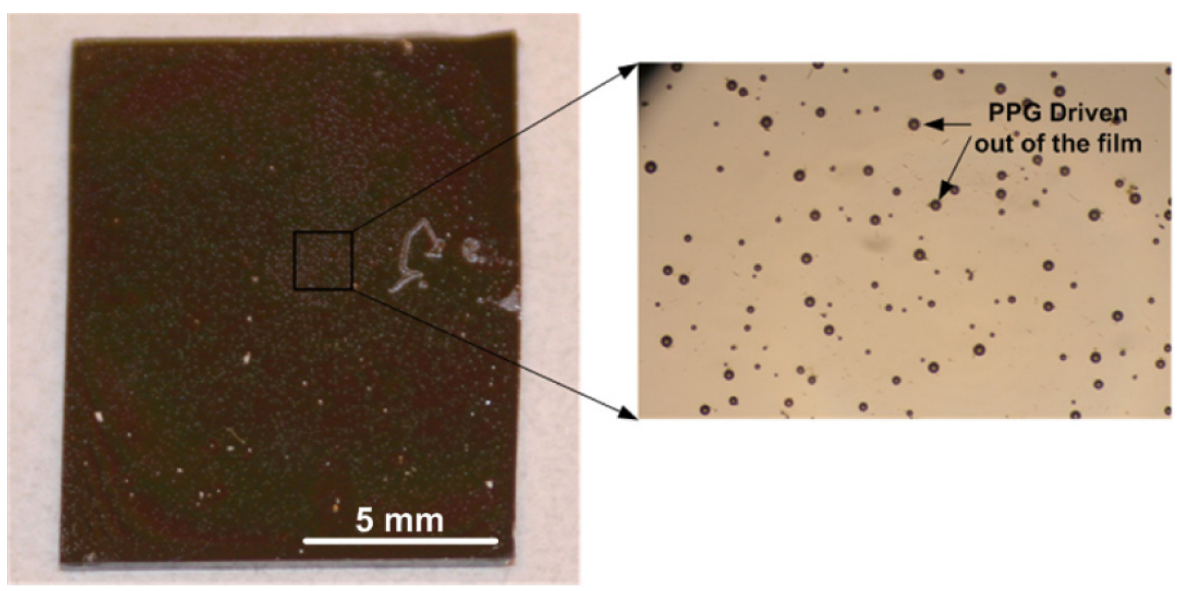

Figure 7. PMSSQ nanoparticle-PPG system behavior at low temperatures. The films were deposited on hydrogen terminated silicon substrates and stored at room temperature $>12 \mathrm{~h}$ before imaging. (The magnified image on the right was taken with an optical microscope with $10 \times$ magnification.)

Table 1. Surface energy dependent porosity of the films following curing/calcination. Surface energies were calculated using the method described in [6]. All the films were cured/calcinated in a pre-heated furnace at $470{ }^{\circ} \mathrm{C}$ for $5 \mathrm{~min}$.

\begin{tabular}{llll}
\hline Sample & $\begin{array}{l}\text { Surface free } \\
\text { energy } \\
\left(\mathrm{mJ} \mathrm{cm}^{-2}\right)\end{array}$ & $\begin{array}{l}\text { Film characteristics } \\
\text { following } \\
\text { curing/calcination }\end{array}$ & $\begin{array}{l}\text { Refractive } \\
\text { Index }\end{array}$ \\
\hline HF dipped silicon & 46.47 & Porous film formation & 1.18 \\
SiCON-silicon & 35.26 & Porous film formation & 1.17 \\
SiCON-glass & 35.90 & Porous film formation & 1.17 \\
HMDS treated glass & 28.03 & Dewetting of film takes place & - \\
Piranha treated glass & 56.93 & Complete pore collapse occurs & 1.36 \\
\hline
\end{tabular}

subsequent covalent bonding to the substrate and the eventual formation of a dense film. Table 1 summarizes the different surfaces used for the film deposition, their corresponding surface energies and the refractive index of the films obtained upon curing/calcination at $500{ }^{\circ} \mathrm{C} / 5 \mathrm{~min}$ in a pre-heated furnace. It should be noted that the porous film formation is indeed not dependent on the thermal conductivity properties of the substrates, as the film prepared on glass substrates retained the porosity with the appropriate surface treatment (see table 1). Dewetting and island formation of the films was observed when the films were deposited on low surface energy substrates (HMDS treated glass substrate). From table 1, it is evident that continuous porous film formation occurs when the films are deposited and heat cured/calcined upon substrates whose surface energies lie between 35 and $47 \mathrm{~mJ} \mathrm{~cm}^{-2}$. This behavior of the system necessitates appropriate treatment of the substrate surfaces to obtain the required porous films. A detailed study on this surface energy dependent behavior will be presented in a subsequent communication.

\section{Conclusion}

We report a simple, spin-on approach for the rapid fabrication of highly porous nanoparticulate films. Nanoparticle-polymer systems at high temperatures have been investigated and the entropic gains experienced by the nanoparticles above the decomposition temperatures of the polymer have been exploited to fabricate these films. Optical properties of these films could be tuned by controlling the curing/calcination temperature or by the poor solvent content in the pre-film formulation. Hydrophobic films with refractive indices as low as 1.047 have been obtained following this approach. The properties of the film greatly depend on the surface energies of the substrate it is deposited upon. Substrates capable of readily interacting with the nanoparticles have been shown to result in complete loss of porosity for the films. This behavior could be conveniently exploited to achieve rapid photopatterning of a pre-cured film that will result in materials with patterned nanoporosity.

\section{Acknowledgments}

We gratefully acknowledge the financial support from National Institute of Health (Award number 2-U42RR014821) and the US army. We thank Lou Ross from the UMC electron microscopy core for his assistance with the SEM images. In addition, we thank Dr Dana D Dlott (University of Illinois, Urbana Champagne), Dr Oomman Varghese (Pennsylvania State University) and Dr Oren Regev (Ben-Gurion University of the Negev, Israel) for their useful comments and discussion.

\section{References}

[1] Balazs A C, Emrick T and Russell T P 2006 Nanoparticle polymer composites: where two small worlds meet Science 314 1107-10 
[2] Baskaran S, Liu J, Domansky K, Kohler N, Li X, Coyle C, Fryxell G E, Thevuthasan S and Williford R E 2000 Low dielectric constant mesoporous silica films through molecularly templated synthesis Adv. Mater. 12 291-4

[3] Biswas K, Gangopadhyay S, Kim H-C and Miller R D 2006 Nanoporous organosilicate films as antireflection coatings Thin Solid Films 514 350-4

[4] Brinker C J, Lu Y, Sellinger A and Fan H 1999 Evaporation-induced self-assembly: nanostructures made easy Adv. Mater. 11 579-85

[5] Chiu J J, Kim B J, Kramer E J and Pine D J 2005 Control of nanoparticle location in block copolymers J. Am. Chem. Soc. 127 5036-7

[6] de Meijer M, Haemers S, Cobben W and Militz H 2000 Surface energy determinations of wood: comparison of methods and wood species Langmuir 16 9352-9

[7] Gupta S, Zhang Q, Emrick T, Balazs A C and Russell T P 2006 Entropy-driven segregation of nanoparticles to cracks in multilayered composite polymer structures Nat. Mater. 5 229-33

[8] Harth E, Horn B V, Lee V Y, Germack D S, Gonzales C P, Miller R D and Hawker C J 2002 A facile approach to architecturally defined nanoparticles via intramolecular chain collapse J. Am. Chem. Soc. 124 8653-60

[9] Jackie Y Y 2000 Nanostructural tailoring: opportunities for molecular engineering in catalysis AIChE J. 46 1902-6

[10] Kim H C, Wilds J B, Kreller C R, Volksen W, Brock P J, Lee V Y, Magbitang T, Hedrick J L, Hawker C J and Miller R D 2002 Fabrication of multilayered nanoporous poly(methyl silsesquioxane) Adv. Mater. 14 1637-9

[11] Ko H, Chang S and Tsukruk V V 2009 Porous substrates for label-free molecular level detection of nonresonant organic molecules ACS Nano 3 181-8

[12] Konjhodzic D, Schröter S and Marlow F 2007 Ultra-low refractive index mesoporous substrates for waveguide structures Phys. Status Solidi a 204 3676-88

[13] Krishnan R S, Mackay M E, Duxbury P M, Hawker C J, Asokan S, Wong M S, Goyette R and Thiyagarajan P 2007 Improved polymer thin-film wetting behavior through nanoparticle segregation to interfaces J. Phys.: Condens. Matter 19356003

[14] Krishnan R S, Mackay M E, Duxbury P M, Pastor A Hawker C J, VanHorn B, Wong M S and Asokan S 2007 Self-assembled multilayers of nanocomponents Nano Lett. 7 484-9

[15] Kucheyev S O et al 2007 Structure of low-density nanoporous dielectrics revealed by low-vacuum electron microscopy and small-angle X-ray scattering Langmuir 23 353-6

[16] Lee B, Park Y-H, Hwang Y-T, Oh W, Yoon J and Ree M 2005 Ultralow-k nanoporous organosilicate dielectric films imprinted with dendritic spheres Nat. Mater. 4 147-50

[17] Lee J Y, Buxton G A and Balazs A C 2004 Using nanoparticles to create self-healing composites J. Chem. Phys. $1215531-40$

[18] Lin Y et al 2005 Self-directed self-assembly of nanoparticle/copolymer mixtures Nature 434 55-9

[19] Maex K, Baklanov M R, Shamiryan D, lacopi F, Brongersma S H and Yanovitskaya Z S 2003 Low dielectric constant materials for microelectronics J. Appl. Phys. 93 8793-841

[20] Oh W and Ree M 2004 Anisotropic thermal expansion behavior of thin films of polymethylsilsesquioxane, a spin-on-glass dielectric for high-performance integrated circuits Langmuir 20 6932-9

[21] Oh W, Shin T J, Ree M, Jin M Y and Char K 2001 Residual stress behavior in methylsilsesquioxane-based dielectric thin films Mol. Cryst. Liq. Cryst. 371 397-402

[22] Othman M T, Lubguban J A, Lubguban A A, Gangopadhyay S, Miller R D, Volksen W and Kim H C 2006 Characterization of porous low-k films using variable angle spectroscopic ellipsometry J. Appl. Phys. 99083503

[23] Rajagopalan T et al 2003 Supercritical carbon dioxide extraction of porogens for the preparation of ultralow-dielectric-constant films Appl. Phys. Lett. 82 4328-30

[24] Raos G and Allegra G 1997 Macromolecular clusters in poor-solvent polymer solutions J. Chem. Phys. 107 6479-90

[25] Ree M, Yoon J and Heo K 2006 Imprinting well-controlled closed-nanopores in spin-on polymeric dielectric thin films J. Mater. Chem. 16 685-97

[26] Risk W, Kim H, Miller R, Temkin H and Gangopadhyay S 2004 Optical waveguides with an aqueous core and a low-index nanoporous cladding Opt. Express 12 6446-55

[27] Rosenholm J M, Meinander A, Peuhu E, Niemi R, Eriksson J E, Sahlgren C and Lindén M 2009 Targeting of porous hybrid silica nanoparticles to cancer cells ACS Nano 3 197-206

[28] Schubert E F, Kim J K and Xi J Q 2007 Low-refractive-index materials: a new class of optical thin-film materials Phys. Status Solidi b 244 3002-8

[29] Thompson R B, Ginzburg V V, Matsen M W and Balazs A C 2001 Predicting the mesophases of copolymer-nanoparticle composites Science 292 2469-72

[30] Tyagi S, Lee J Y, Buxton G A and Balazs A C 2004 Using nanocomposite coatings to heal surface defects Macromolecules 37 9160-8

[31] Veres J, Ogier S D, Leeming S W, Cupertino D C and Khaffaf S M 2003 Low-k insulators as the choice of dielectrics in organic field-effect transistors $A d v$. Funct. Mater. 13 199-204

[32] Warren S C, Messina L C, Slaughter L S, Kamperman M, Zhou Q, Gruner S M, DiSalvo F J and Wiesner U 2008 Ordered mesoporous materials from metal nanoparticle-block copolymer self-assembly Science 320 1748-52

[33] Williams C, Brochard F and Frisch H L 1981 Polymer collapse Annu. Rev. Phys. Chem. 32 433-51

[34] Yabu H, Higuchi T, Ijiro K and Shimomura M 2005 Spontaneous formation of polymer nanoparticles by good-solvent evaporation as a nonequilibrium process Chaos: Interdiscip. J. Nonlinear Sci. 15047505

[35] Yang C-C, Wu P-T, Chen W-C and Chen H-L 2004 Low dielectric constant nanoporous poly(methyl silsesquioxane) using poly(styrene-block-2-vinylpyridine) as a template Polymer 45 5691-702 